\title{
DETERMINAÇÃO DE RESÍDUOS DE ORGANOCLORADOS NO LEITE EM PÓ DISTRIBUÍDO EM PIRACICABA (SP)
}

\author{
ROSANA MARIA OLIVEIRA FREGUGLIA* \\ GRAZIELA CRISTINA ROSSI MOURA-ANDRADE** \\ VALDEMAR LUIZTORNISIELO***
}

\begin{abstract}
No presente trabalho foi determinada a presença de resíduos dos organoclorados hexaclorobenzeno, lindane, heptacloro, aldrin, heptacloro epóxi, o, $p^{\prime}$ DDE, á-endosulfan, $p, p^{\prime}$ DDE, dieldrin, endrin, âendosulfan, endosulfan sulfato e metoxicloro em amostras de leite em pó integral distribuído pelo programa municipal, na cidade de Piracicaba (SP). Após a extração das amostras com cartuchos $\mathrm{C}_{18} \mathrm{e}$ câmara de vácuo, realizou-se a identificação e quantificação dos resíduos de organoclorados por cromatografia a gás utilizando detector de captura de elétrons (CG/DCE) e confirmação mediante espectrometria de massas (CG/EM). Observou-se a contaminação de uma amostra por heptacloro, abaixo do Limite Máximo de Resíduo Estranho estabelecido pelo Codex Alimentarius. Esse fato é preocupante devido à importância do leite na alimentação infantil e porque o heptacloro não é permitido em amostras de leite.
\end{abstract}

PALAVRAS-CHAVE: LEITE EM PÓ; RESÍDUOS DE PESTICIDAS; PESTICIDAS; ORGANOCLORADOS.

* Doutora em Ciências, Universidade de São Paulo (USP), Centro de Energia Nuclear na Agricultura, Departamento de Ecotoxicologia, Piracicaba, SP (ro_freguglia@hotmail.com).

** Doutoranda em Ciências, USP, Centro de Energia Nuclear na Agricultura, Departamento de Ecotoxicologia, Piracicaba, SP (grazielacrm@yahoo.com.br).

*** Doutor em Tecnologia Nuclear, Professor, USP, Centro de Energia Nuclear na Agricultura, Departamento de Ecotoxicologia, Piracicaba, SP (vltornis@cena.usp.br). 


\section{INTRODUÇÃO}

O controle químico de pragas reduziu o índice de doenças e aumentou a produção agrícola, mas esses compostos químicos podem permanecer ativos no meio ambiente por longos períodos, afetando os ecossistemas. Os efeitos desses compostos ao longo do tempo representam grande risco para a saúde pública, sendo necessários o monitoramento e a vigilância desses produtos em águas, solos, alimentos e ar (LARA e BATISTA, 1992; ARAÚJO, NOGUEIRA e AUGUSTO, 2000).

Os compostos organoclorados (OC) foram muito utilizados e ainda são em alguns países com propósitos agrícolas e principalmente sanitários, mas devido à sua estabilidade química, persistência e seu caráter lipofílico podem se acumular em tecido adiposo. A combinação entre a baixa solubilidade em água e a alta capacidade de adsorção na matéria orgânica leva ao acúmulo desses compostos ao longo da cadeia alimentar, especialmente nos tecidos ricos em gorduras dos organismos vivos (TORRES, 1998). Vários estudos têm sido realizados sobre a ocorrência dos OC no leite humano, evidenciando o mau uso e a consequente acumulação (SALEH et al., 1996; MATUO et al., 1992; SALEM, AHMAD e ESTAITIEH, 2009; )

A exposição humana aos organoclorados ocorre principalmente mediante alimentos contaminados, em especial pela ingestão de produtos animais. O risco potencial desses compostos é estimado a partir de estudos experimentais, não tendo sido ainda documentados seus efeitos diretos na saúde humana com base em alterações celulares. Vários desses compostos revelaram-se capazes de induzir ou promover câncer de mama experimentalmente (ADAMI et al., 1995).

No Brasil, a Portaria no 329 de 02 de setembro de 1985 proibiu em todo território nacional, a comercialização, o uso e a distribuição dos produtos agrotóxicos organoclorados, destinados à agropecuária, sendo revogada pela Portaria $n^{\circ} 424$ (de 07 de novembro de 1985) a permissão de sua comercialização, uso e distribuição em caráter emergencial a critério da Secretaria Nacional de Defesa Agropecuária do Ministério da Agricultura (BRASIL, 1985). Embora a proibição esteja em vigor desde 1985, ainda são encontrados resíduos desses compostos em diversas matrizes (ANVISA, 2007)

Constituído basicamente de lactose, gordura, proteínas e sais minerais, o leite é rico em vitaminas A, B e D. A vitamina A fortalece a visão e ajuda a combater infecções, a vitamina B favorece o crescimento normal da criança, sendo essencial para a saúde da pele e a vitamina $D$ ajuda no crescimento normal do esqueleto, evitando o raquitismo, pois é indispensável para a absorção de cálcio e fósforo. Devido à sua composição, o leite é de extrema importância na alimentação infantil (INMETRO, 2010).

O leite em pó, produzido a partir do leite integral pasteurizado, sofre deterioração mais rápida que o leite. É classificado de acordo com o tratamento térmico que recebe, comumente efetuado com base na medição da quantidade de proteínas do soro que sobram no pó após esse tratamento (KELTS e JONES, 1995).

As exportações mundiais de leite em pó integral foram estimadas em 1.826 mil toneladas em 2009 , com queda de 1,2\% em relação a 2008, principalmente devido à previsão de diminuição das cotas de exportação da União Européia em 100 mil toneladas. O leite em pó integral constitui produto chave de exportação para as regiões produtoras com leite excedente, principalmente para mercados importadores de países em desenvolvimento (FAO, 2009).

O Brasil importa leite em pó principalmente da Argentina, através do Mercosul e, para isto, houve necessidade de ajustes na legislação. Os pontos já harmonizados no Mercosul sobre o estabelecimento de níveis máximos de contaminantes químicos em alimentos foram resolvidos pela Portaria $n^{\circ}$ 685, de 27 de agosto de 1998 e sugerem a utilização das normas, diretrizes ou recomendações da Comissão do Codex Alimentarius, da União Européia, da Food and Drug Administration ou outros organismos reconhecidos internacionalmente para o estabelecimento de níveis máximos de resíduos de pesticidas (BRASIL, 1998). Na Argentina, o Instituto Nacional de 
Alimentos realiza análises de pesticidas organoclorados e organofosforados em alimentos (ANMAT, 2010).

Existem poucos relatos e estudos sobre a determinação de pesticidas em amostras de leite em pó no Brasil. Em 2002, a Agência Nacional de Vigilância Sanitária (ANVISA) criou o Programa de Análise de Resíduos de Medicamentos Veterinários em Alimentos de Origem Animal, conforme a RDC nº. 253 de 16 de setembro de 2003, que determina os Limites Máximos de Resíduos (LMR) de antimicrobianos, micotoxinas, antiparasitários e organoclorados em amostras de leite e leite em pó. Esse programa veio somar-se ao Programa de Análise de Resíduos de Agrotóxicos (PARA) ampliando, assim, a capacidade do Governo em promover medidas preventivas de controle para melhorar o controle sanitário dos alimentos (ANVISA, 2006).

O presente estudo teve por objetivo avaliar níveis de pesticidas organoclorados em leite em pó, distribuído pelos programas estadual e federal, que atendem crianças carentes da região de Piracicaba (São Paulo).

\section{MATERIAL E MÉTODOS}

As análises foram conduzidas no Laboratório de Ecotoxicologia do Centro de Energia Nuclear na Agricultura (CENA/USP), em Piracicaba (SP).

\subsection{AMOSTRAGEM}

As amostras foram fornecidas pelo Sistema de Vigilância de Avaliação Nutricional (SISVAN), ligado à Secretaria Municipal de Saúde de Piracicaba, SP. O leite em pó destina-se a famílias carentes cadastradas junto ao programa dos governos estadual (Vivaleite) e federal, sendo distribuído pela Prefeitura Municipal (Secretaria da Saúde) para atender crianças de 06 meses até 2 anos. Como a cidade de Piracicaba é atendida pelos programas federal e estadual foram analisadas as duas marcas recebidas pela prefeitura, denominadas de $\mathrm{X}$ e $\mathrm{Y}$, respectivamente.

$\mathrm{O}$ leite em pó identificado com as marcas $\mathrm{X}$ e $\mathrm{Y}$ foi embalado no Brasil, mas trata-se de leite importado da região de Buenos Aires (Argentina). O leite em pó da marca $Y$ foi classificado como leite em pó integral instantâneo enriquecido com vitaminas e ferro, e o da marca $X$ como leite em pó integral.

\subsection{SOLUÇÕES PADRÃO}

Diluiu-se a solução estoque contendo a mistura de padrões ou "mix" de pesticidas organoclorados (ChemService, West Chester, USA) em hexano grau resíduo (Lichrosolv). A partir dessa solução foram diluídas soluções de trabalho nas concentrações: 0,$01 ; 0,02 ; 0,03 ; 0,04 ; 0,05 \eta g \mu \mathrm{L}^{-1}$ em hexano.

\subsection{ORGANOCLORADOS ESTUDADOS}

Foram rastreados os seguintes organoclorados: hexaclorobenzeno, lindane, heptacloro, aldrin, heptacloro epóxi, $o, p^{\prime}$ DDE, endosulfan I, $p, p^{\prime}$ DDE, dieldrin, endrin, endosulfan II, endosulfan sulfato e metoxicloro.

\subsection{PREPARAÇÃO DAS AMOSTRAS E DETERMINAÇÕES ANALÍTICAS}

As amostras foram mantidas em freezer, sob congelamento até o processamento, sendo o leite em pó reconstituído de acordo com a especificação do fabricante, com água destilada. Foram preparados $200 \mathrm{~mL}$ de cada amostra, usando-se $15 \mathrm{~g}$ de leite em pó para cada $100 \mathrm{~mL}$ de água. Três 
repetições de cada amostra foram analisadas, determinando-se cada repetição em triplicata. Para o Branco, seguiu-se o procedimento de extração, utilizando somente os solventes envolvidos na extração. Como testemunha utilizou-se amostra de leite sem fortificação (sem adição do padrão).

Empregaram-se na extração, cartuchos $C_{18}$ (Sep-Pak Vac - 1 g, Waters Corporation) com capacidade de $6 \mathrm{~mL}$ e câmara de vácuo, conforme procedimento descrito por Mañes, Font e Picó (1993) e utilizado por Picó et al. (1995) para determinação de organoclorados em formulações infantis.

Para a quantificação dos organoclorados foi utilizado cromatógrafo a gás Chrompack CP 9001, equipado com detector de captura de elétrons ${ }^{63} \mathrm{Ni}$, injetor "split" e nitrogênio ultrapuro como gás de arraste.

Utilizou-se coluna DB-5ms (J\&W Scientific Inc.) com temperatura inicial de $240^{\circ} \mathrm{C}$ e final de $280^{\circ} \mathrm{C}$, com taxa de aquecimento de $20^{\circ} \mathrm{C}$ minuto ${ }^{-1}$. A temperatura do injetor foi de $250^{\circ} \mathrm{C}$ e a do detector de $300^{\circ} \mathrm{C}$, com fluxo do make-up $50 \mathrm{~mL} \mathrm{~min}^{-1} \mathrm{e}$ volume de injeção de $2 \mu \mathrm{L}$. O tempo total da corrida foi de 15 minutos.

Para a confirmação dos organoclorados nas amostras que se apresentaram contaminadas utilizou-se Cromatógrafo a gás acoplado a espectrômetro de massa (CG/EM) Finnigan CGQ, nas seguintes condições: temperatura inicial de $220^{\circ} \mathrm{C}$ por 2 minutos, taxa de aquecimento de $20^{\circ} \mathrm{C}$ minuto $^{-1}$ até $240^{\circ} \mathrm{C}$ por 8 minutos, aumento de $20^{\circ} \mathrm{C}$ minuto ${ }^{-1}$ até $280^{\circ} \mathrm{C}$ por 2 minutos, temperatura do injetor de $250^{\circ} \mathrm{C}$; injeção splitless, fluxo da $1 \mathrm{~mL} \mathrm{~min}^{-1}$ (Hélio como gás de arraste) e modo full scan. O tempo total da corrida foi de 13 minutos.

\subsection{VALIDAÇÃO}

A linearidade é a habilidade de um método analítico em produzir resultados diretamente proporcionais à concentração do analito nas amostras em dada faixa de concentração (INMETRO, 2007). As curvas analíticas foram obtidas por padronização externa, relacionando-se a área do pico versus a concentração $\left(n g \mu \mathrm{L}^{-1}\right)$ nas concentrações de 0,01 a 0,05 $\eta \mathrm{g} \mu \mathrm{L}^{-1}$.

Os limites de detecção foram determinados para os diferentes organoclorados da mistura de padrões, injetando-se diluições com diferentes concentrações. Definiu-se o limite de detecção (expresso por $\eta g \mu \mathrm{L}-1$ ) como a menor concentração da substância em exame que pode ser detectada, mas não necessariamente quantificada, utilizando-se determinado procedimento experimental.

Avaliou-se a exatidão do método mediante ensaios de recuperação. As amostras de leite foram fortificadas com os organoclorados nas concentrações lineares de 0,01 a 0,05 $\eta \mathrm{gL} \mathrm{L}^{-1}$, homogeneizadas e colocadas a $4^{\circ} \mathrm{C}$ "overnight". Os valores aceitos para ensaios de recuperação para a avaliação do método analítico encontram-se entre 70 e 120\% e o coeficiente de variação (precisão) não deve exceder a $20 \%$.

\section{RESULTADOS E DISCUSSÃO}

Os resultados foram avaliados de acordo com o Codex Alimentarius (FAO, 2010), comparandoos com os valores dos Limites Máximos de Resíduos (LMR) e Limites Máximos de Resíduos Estranhos (LMRE), respectivamente. Os LMR apresentados no CODEX representam os limites aceitáveis de pesticidas e tem por objetivo facilitar o comércio internacional de alimentos e proteger a saúde dos consumidores. Os LMRE se referem aos resíduos de pesticidas encontrados em alimentos, mas seu uso não é autorizado para aquela cultura pela legislação vigente, ou não há registro do pesticida naquele país.

Os valores dos ensaios de recuperação, apresentados na Tabela 1, estão de acordo com os critérios estabelecidos para validação de métodos analíticos empregados para análise de resíduos de pesticidas em alimentos. 


\section{TABELA 1 - PORCENTAGEM DE RECUPERAÇÃO $\left(\% R_{m}\right)$, DESVIO-PADRÃO $(\sigma)$ E COEFICIENTE DE VARIAÇÃO (CV\%) OBTIDO APÓS TRÊS EXTRAÇÕES DOS PESTICIDAS EM LEITE EM PÓ}

\section{Organoclorado}

\section{Fortificação $\left(\eta g \mu \mathrm{L}^{-1}\right)$}

\begin{tabular}{|c|c|c|c|c|c|c|c|c|c|}
\hline & \multicolumn{3}{|c|}{0,01} & \multicolumn{3}{|c|}{0,03} & \multicolumn{3}{|c|}{0,05} \\
\hline & $\% R_{m}{ }^{a}$ & $\sigma^{b}$ & $\mathrm{CV}^{\mathrm{c}}$ & $\% R_{m}{ }^{a}$ & $\sigma^{b}$ & $\mathbf{C V}^{\mathrm{c}}$ & $\% R_{m}$ & $\sigma^{b}$ & $\mathrm{CV}^{\mathrm{c}}$ \\
\hline Hexaclorobenzeno & 99,85 & 1,87 & 1,87 & 102,21 & 0,98 & 0,96 & 99,28 & 1,18 & 1,18 \\
\hline Lindane & 80,16 & 1,39 & 1,73 & 105,13 & 1,32 & 1,17 & 89,09 & 1,25 & 1,40 \\
\hline Heptacloro & 98,54 & 2,74 & 2,78 & 101,82 & 1,98 & 1,94 & 97,73 & 2,52 & 2,58 \\
\hline Aldrin & 84,31 & 1,22 & 1,45 & 91,70 & 0,99 & 1,08 & 99,24 & 0,98 & 0,99 \\
\hline Heptacloro epóxi & 106,11 & 1,86 & 1,75 & 102,87 & 2,23 & 2,17 & 101,22 & 0,96 & 0,95 \\
\hline$o, p^{\prime} \mathrm{DDE}$ & 99,00 & 0,89 & 0,90 & 92,18 & 1,52 & 1,65 & 92,77 & 1,78 & 1,92 \\
\hline Endosulfan I & 84,30 & 3,22 & 3,82 & 103,28 & 1,75 & 1,69 & 100,63 & 1,56 & 1,55 \\
\hline$p, p^{\prime} \mathrm{DDE}$ & 86,60 & 0,59 & 0,59 & 106,92 & 2,11 & 1,97 & 96,33 & 0,90 & 0,90 \\
\hline Dieldrin & 84,09 & 1,27 & 1,51 & 99,41 & 1,95 & 1,96 & 96,12 & 2,52 & 2,62 \\
\hline Endrin & 89,38 & 2,47 & 2,76 & 94,70 & 2,15 & 2,27 & 97,65 & 2,53 & 2,59 \\
\hline Endosulfan II & 85,49 & 2,20 & 2,57 & 88,21 & 2,10 & 2,38 & 98,52 & 1,87 & 1,90 \\
\hline Endosulfan sulfato & 78,54 & 2,83 & 3,60 & 88,89 & 1,85 & 2,08 & 99,48 & 1,96 & 1,97 \\
\hline Metoxicloro & 111,41 & 1,67 & 1,50 & 88,73 & 1,96 & 2,21 & 101,87 & 0,98 & 0,96 \\
\hline
\end{tabular}

A otimização dos parâmetros cromatográficos referentes à programação de temperatura favoreceu a separação dos picos da solução de mistura de padrões.

Dos organoclorados rastreados, somente o heptacloro foi identificado e quantificado na amostra Y-02 com concentração de $0,003 \mathrm{mg} \mathrm{kg}^{-1}$ (CV de 5\%), porém abaixo dos limites estabelecidos pela FAO/CODEX (2010). Para a confirmação dos organoclorados encontrados na amostra foi utilizada a cromatografia a gás acoplada à espectrometria de massas (CG-EM). A Figura 1 apresenta o cromatograma dos organoclorados: hexaclorobenzeno, lindane, heptacloro, aldrin, heptacloro epóxi, $o, p^{\prime} \mathrm{DDE}$, endosulfan I, $p, p^{\prime} \mathrm{DDE}$, dieldrin, endrin, endosulfan II, endosulfan sulfato e metoxicloro, nessa sequência, na concentração de $0,03 \mathrm{~g} \mu \mathrm{L}^{-1} \mathrm{em}$ hexano.

As amostras de leite em pó analisadas atenderam às determinações exigidas pelo CODEX, porém o organoclorado heptacloro não deveria estar presente em leite em pó, mesmo em concentrações inferiores ao LMR permitido. O leite constitui um dos principais componentes da nutrição infantil, podendo ser introduzido na alimentação a partir dos 6 meses de idade.

A direção do Sindicato da Indústria de Laticínios e Produtos derivados do Estado de Minas Gerais (SILEMG), preocupada com a qualidade do leite em pó consumido no Brasil, solicitou ao Inmetro a inserção do leite em pó integral no Programa de Análise da Qualidade dos Produtos em 1998. Os resultados obtidos pelo Inmetro demonstraram problemas, principalmente, em relação ao teor de lipídios nas 9 marcas nacionais e 3 importadas analisadas. Isso não afeta a saúde do consumidor, porém não foram realizadas análises de contaminantes orgânicos (INMETRO, 2010). 


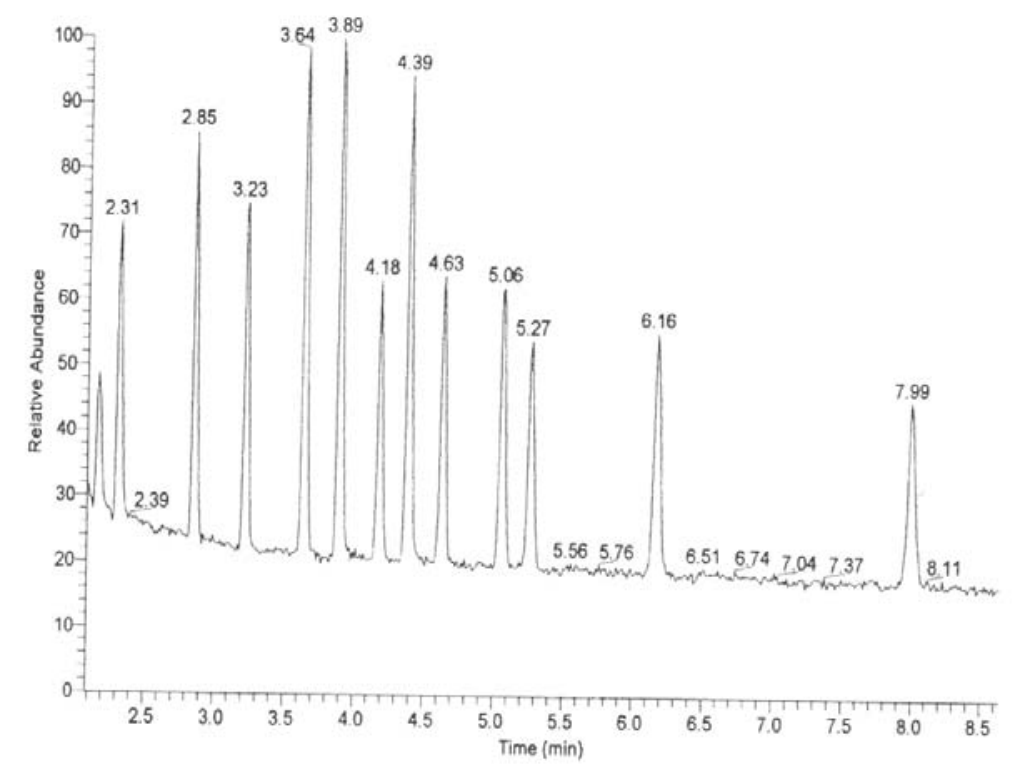

\section{FIGURA 1 - CROMATOGRAMA PARA CONFIRMAÇÃO DOS PESTICIDAS ANALISADOS POR CG-EM NA CONCENTRAÇÃO $0,03 \eta g \mu \mathrm{L}^{-1}$ EM HEXANO}

São poucas as pesquisas realizadas nesse tipo de matriz no Brasil, sendo geralmente as análises feitas por órgãos públicos credenciados com o intuito de fixar a identidade e as características mínimas de qualidade do leite em pó destinado ao consumo humano. A partir de 1998, depois de alguns incidentes internacionais e restrições à importação de produtos lácteos brasileiros por alguns órgãos internacionais, principalmente o mercado europeu, a ANVISA intensificou seu controle sobre o produto.

\section{CONCLUSÃO}

O controle de qualidade deve ser considerado como a principal ação preventiva de saúde pública com relação à contaminação de alimentos, e o monitoramento deve ser constante, promovendo à população acesso a alimentos seguros, significando menores índices de doenças de origem alimentar, menores custos para a saúde pública, além da diminuição das barreiras ao comércio internacional.

A importância do leite em pó na alimentação infantil requer atenção especial nos programas de monitoramento, visando à proteção e contribuição para o desenvolvimento integral da criança.

As medidas restritivas ou proibitivas, tomadas nos últimos anos pelos governos com relação ao monitoramento de resíduos de pesticidas e a qualidade dos alimentos, representam grande passo na tentativa de diminuir o impacto negativo dos pesticidas na segurança alimentar. No entanto, outras medidas são necessárias para que a situação não se agrave ainda mais. A evidência da contaminação em amostra de leite em pó por pesticidas organoclorados justifica estudos mais frequentes junto aos programas de distribuição desse alimento.

\section{ABSTRACT}

\section{DETERMINATION OF ORGANOCHLORINE RESIDUES IN MILK POWDER DISTRIBUTED IN PIRACICABA, SP (BRAZIL)}

In the present study was determined the presence of organochlorine residues hexachlorobenzene, lindane, 
heptachlor, aldrin, heptachlor epoxide, o, p 'DDE, á-endosulfan, p, p’ DDE, dieldrin, endrin, â-endosulfan, endosulfan sulfate and methoxychlor in milk powder samples distributed by the municipal program in Piracicaba, SP (BRAZIL). After extraction of samples with C18 cartridges and a vacuum was performed to identificate and quantificate residues of organochlorine compounds by gas chromatography using electron capture detector (GC/ECD) and confirmation using mass spectrometry (GC/MS). It was observed the heptachlor contamination in one sample, below the levels established by Extraneous Maximum Residue Limits determined by the Codex Alimentarius, but due to its importance in infant feeding this fact is alarming, because the heptachlor is not allowed in milk samples.

KEY-WORDS: MILK POWDER; PESTICIDES - RESIDUES; ORGANOCHLORINE.

\section{REFERÊNCIAS}

1 ADAMI, H.O.; LIPWORTH, L.; TITUS-ERNSTOFF, L.; HSIEH, C.C.; HAMBERG, A.; AHLBORG, U.; BARON, J. Organochlorine compounds and estrogen related cancers in women. Cancer Causes Control, v.6, p.551-566, 1995.

2 ANMAT. Administración Nacional de Medicamentos, Alimentos y Tecnología Médica. Departamento Control y Desarrollo. Análisis de alimentos, 2010. Disponível em: < http://www.anmat.gov.ar/formularios/alimentos/servicios.asp>. Acesso em: 23/02/2010.

3 ANVISA. Agência Nacional de Vigilância Sanitária. Agrotóxicos e toxicologia. Brasília, 2007. Disponível em: < http://www.anvisa.gov.br/toxicologia/residuos/ anexo_relatorio_2001_2007.pdf >. Acesso em: 23/02/2010.

4 ANVISA. Agência Nacional de Vigilância Sanitária. Programa de Análise de resíduos de medicamentos veterinários em alimentos de origem animal. PAMVet - Relatório 2004/2005. Brasília, 2006. Disponível em: < http://portal.anvisa.gov.br/wps/wcm/connect/90236e804004751bad24afee27e7f6ac/relatorio_leite_200405.pdf?MOD=AJPERES> Acesso em: 25/02/2010.

5 ARAÚJO, A.C.P.; NOGUEIRA, D.P.; AUGUSTO, L.G.S. Impacto dos praguicidas na saúde: estudo da cultura do tomate. Revista de Saúde Pública, São Paulo, v. 34, n.3, p.309-313, 2000.

6 BRASIL. Ministério da Agricultura, Pecuária e Abastecimento. Portaria n 424, de 07 de novembro de 1985. Dispõe sobre a proibição, em todo território nacional, a comercialização, o uso e a distribuição dos produtos agrotóxicos organoclorados, destinados à agropecuária, dentre outros. Disponível em: < http:// extranet.agricultura.gov.br/sislegis-consulta/consultarLegislacao.do? operacao=visualizar\&id=7320 $>$. Acesso em: 23 fev. 2010.

7 BRASIL. Ministério da Saúde. Secretaria de Vigilância Sanitária. Portaria nº 685, de 27 de agosto de 1998. Dispõe sobre a aprovação do Regulamento técnico: "princípios gerais para o estabelecimento de níveis máximos de contaminantes químicos em alimentos" e seu anexo: "limites máximos de tolerância para contaminantes inorgânicos". Disponível em: < http://www.anvisa.gov.br/legis/portarias/685_98.htm>. Acesso em: 23/02/2010.

8 FAO. Food and Agriculture Organization. Milk and milk products. Production, 2009. Disponível em: < http:// www.fao.org/docrep/011/ai482e/ai482e09.htm>. Acesso em: 23/02/10.

9 FAO/WHO. Food and Agriculture Organization/World Health Organization. Codex Alimentarius: pesticide residues in food. 2010. Disponível em: <http://www.codexalimentarius.net/mrls/pestdes/jsp/pest_q-e.jsp>. Acesso em: $12 / 01 / 2010$.

10 INMETRO. Instituto Nacional de Metrologia, Normalização e Qualidade Industrial. Orientação sobre validação de métodos de ensaios químicos: DOQ-CGCRE-008. Rev. 02. Rio de Janeiro, 2007. 24 p.

11 INMETRO. Instituto Nacional de Metrologia. Informações ao consumidor. Brasília, 2010. Disponível em:<http:// www.inmetro.gov.br/consumidor/produtos/leitepo.asp>. Acesso em: 23 fev. 2010.

12 KELTS D.G.; JONES, E.G. Manual de nutrição infantil. Rio de Janeiro: Editora Guanabara, 1995. 312 p.

13 LARA, W.H.; BATISTA, G.C. Pesticidas. Química Nova, São Paulo, v.15, n.2, p.161,1992.

14 MAÑES, J.; FONT, G.; PICÓ, Y. Evaluation of a solid-phase extraction system for determining pesticide residues in milk. Journal of Chromatography, v.642, p.195-204, 1993.

15 MATUO, Y.K.; LOPES, J.N.C.; CASANOVA, I.C.; MATUO, T.; LOPES, J.L.C. Organoclhorine pesticide residues in human milk in the Ribeirão Preto region State of São Paulo, Brazil. Archives of Environmental Contamination and Toxicology, v.22, p.167-175, 1992.

16 PICÓ, Y.; VIANA, Z.; FONT, G.; MANES, J. Determination of organochlorine pesticides content in human milk and infant formulas using solid phase extraction and capillary gas chromatography. Journal of the Agricultural and Food Chemistry, v.43, n.6, p.1610-1615, 1995. 
17 SALEH, M; KAMEL, A; RAGAB, A; EL-BAROTY, G; EL-SEBAE, A.K. Regional distribution of organochlorine inseticide residues in human milk from Egypt. Journal Environmental Science Health, v.B31, p.241-255, 1996.

18 SALEM, N. M.; AHMAD, R.; ESTAITIEH, H. Organochlorine pesticide residues in dairy products in Jordan. Chemosphere, v.77, p. 673-678, 2009.

19 TORRES, J.P.M. Ocorrência de micropoluentes orgânicos (organoclorados e hidrocarbonetos policíclicos aromáticos) em sedimentos fluviais e solos tropicais. 1998. 139 p. Tese (Doutorado em Ciências) - Instituto de Biofísica Carlos Chagas Filho, Centro de Ciências da Saúde, Universidade Federal do Rio de Janeiro, Rio de Janeiro, 1998.

\section{AGRADECIMENTOS}

Os autores agradecem à Fundação de Amparo a Pesquisa do Estado de São Paulo (FAPESP) e ao Conselho Nacional de Desenvolvimento Científico e Tecnológico (CNPq) pelo apoio financeiro. 